\title{
Detecting single molecules inside a carbon nanotube to control molecular sequences using inertia trapping phenomenon
}

\author{
Z. L. Hu, ${ }^{1,2}$ Gustaf Mårtensson, ${ }^{1}$ Murali Murugesan, ${ }^{1}$ Yifeng Fu, ${ }^{1}$ Xingming Guo, ${ }^{3}$ \\ and Johan Liu ${ }^{1,3, a)}$ \\ ${ }^{1}$ Chalmers University of Technology, Kemivägen 9, Se 41296 Göteborg, Sweden \\ ${ }^{2}$ Shanghai Institute of Applied Mathematics and Mechanics, No. 149, Yan Chang Road, Shanghai 200072, \\ China \\ ${ }^{3}$ Key Laboratory of New Displays and System Integration, SMIT Center and School of Mechatronics \\ and Mechanical Engineering, Shanghai University, No. 149, Yan Chang Road, Shanghai 200072, China
}

(Received 1 July 2012; accepted 10 September 2012; published online 25 September 2012)

\begin{abstract}
Here we show the detection of single gas molecules inside a carbon nanotube based on the change in resonance frequency and amplitude associated with the inertia trapping phenomenon. As its direct implication, a method for controlling the sequence of small molecule is then proposed to realize the concept of manoeuvring of matter atom by atom in one dimension. The detection as well as the implication is demonstrated numerically with the molecular dynamics method. It is theoretically assessed that it is possible for a physical model to be fabricated in the very near future. (C) 2012 American Institute of Physics. [http://dx.doi.org/10.1063/1.4754617]
\end{abstract}

The manoeuvring of matter atom by atom may be one of the ultimate goals of the science of nanotechnology. ${ }^{1-3}$ This goal was partially realized by the scanning tunnelling microscope (STM) with its ability to manipulate atoms on the surface of a metal. There are a number of different approaches to nanoscale mass transport ${ }^{7-16}$ based on carbon nanotube (CNT) nanoelectromechanical systems (NEMS) that attempt to exhibit promising properties of the CNT. The experimental approaches include electrophoretic, ${ }^{7-9}$ thermophoretic, ${ }^{10,11}$ and mechanically actuated ${ }^{12-16}$ methods. However, all these approaches failed to achieve manoeuvring matter at the atomic level. On the other hand, in spite of fundamental and applied interest in the interaction of CNTs and nanoparticles due to mechanical vibrations of the CNT, ${ }^{17-23}$ little attention has been paid to the manipulation of nanoparticles and nanoscale mass transport based on CNT-enabled NEMS. ${ }^{4-6,12,13}$ The inertia trapping phenomenon ${ }^{4-6}$ which shows that atomic particles in a vibrating CNT have a tendency to move toward the antinodes and stay in the antinodes vicinity, due to inertial forces (or centrifugal forces), is then adopted by this paper to realize the concept of matter manipulation. Although it is not a true full three dimensional (3D) manoeuvring matter at the atomic level, the work could still be valuable in fields like synthetic biology ${ }^{24}$ and molecule level data storage $e^{7,25,26}$ and hopefully a key step to the realization of true 3D nanotechnology. ${ }^{3}$

The encoding of a molecular chain can be realized by shooting several kinds of gas molecules into the CNT one by one as shown in Figure 1. One challenge encountered in the present work was, the detection of molecules inside the CNT required by step 2 as shown in Figure 1, which was resolved using a CNT resonator system. The CNT resonator is able to detect the change of its vibration amplitude by detecting the change of electric current in a circuit and thus detect small changes of its eigenfreqency. ${ }^{17-21}$ More details about this detection technique are given in supplementary information

\footnotetext{
${ }^{\text {a) }}$ Author to whom correspondence should be addressed. Electronic mail: jliu@chalmers.se.
}

Sec. A. ${ }^{30}$ The detection process can then be split into two categories: (a) increasing the vibrational amplitude due to the insertion of the molecules denoted as " + ," and (b) decreasing the amplitude denoted as " - ." For a "+" detection, the resonator is initially away from resonation which causes small amplitude; after the insertion of molecule, it achieves resonation hence larger amplitude was observed. For "-" detection the inverse is happens. The situation can further be divided into two subsets according to the dynamical boundary conditions of the CNT resonator, i.e., the double-clamped CNT denoted as " $\alpha$ " and the free-ended cantilever CNT denoted as " $\beta$." Therefore, 4 types of molecular chain encoders (namely encoder $\alpha+, \alpha-, \beta+$, and $\beta-$ ) were proposed, and we systematically tested all of them. The encoder $\alpha+$ for example is composed of a double-clamped CNT and detects the molecule after detecting an increasing of the amplitude. Note that " $\beta$ " can only encode a finite length of molecular chain due to the restricted length of the cantilever. Therefore, the schematic in Figure 1 requires a little modification for " $\beta$ " encoders by omitting the switch with capped CNT. The resonator itself acts as a storage area for the molecular chain.

A molecular dynamics (MD) simulation is performed to verify if the molecule can remain in the CNT after getting into the resonator and influence the vibrational amplitude. Consistent with a previous study, ${ }^{5}$ a $(5,5)$ CNT and krypton $\mathrm{Kr})$ based monatomic molecules were utilized in the simulations. The detailed simulations are given in the supplementary information Sec. A. ${ }^{30}$ Encoder $\alpha+$ was studied as a start. The molecular model is shown in Figure 2(a). A Kr-atom was then shot into the CNT which was vibrationally excited. Figure 2(b) shows trapping process of the particle after its insertion into the CNT. With details in the supplementary information Sec. $\mathrm{A},{ }^{30}$ we concluded that the amplitude of the CNT resonator vibration increased from $8.80 \AA$ to $8.99 \AA$ as shown in Table I due to the insertion of the Kr-atom, which verifies that the particle has indeed entered the CNT. A further simulation reveals that if the excitation stops, the 

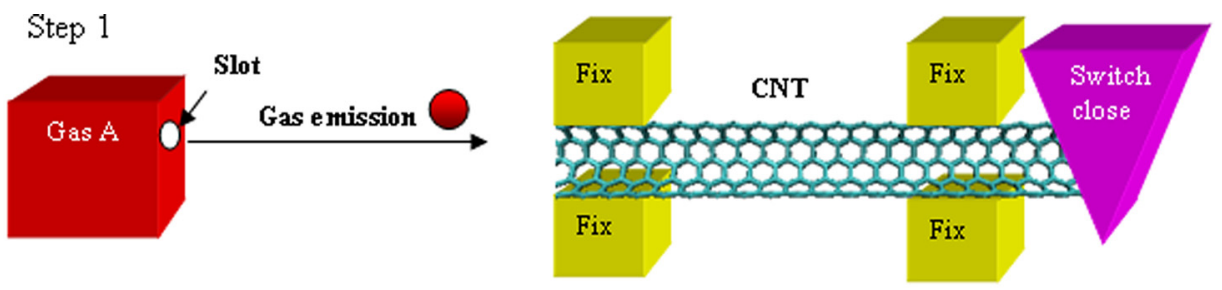

Step 2
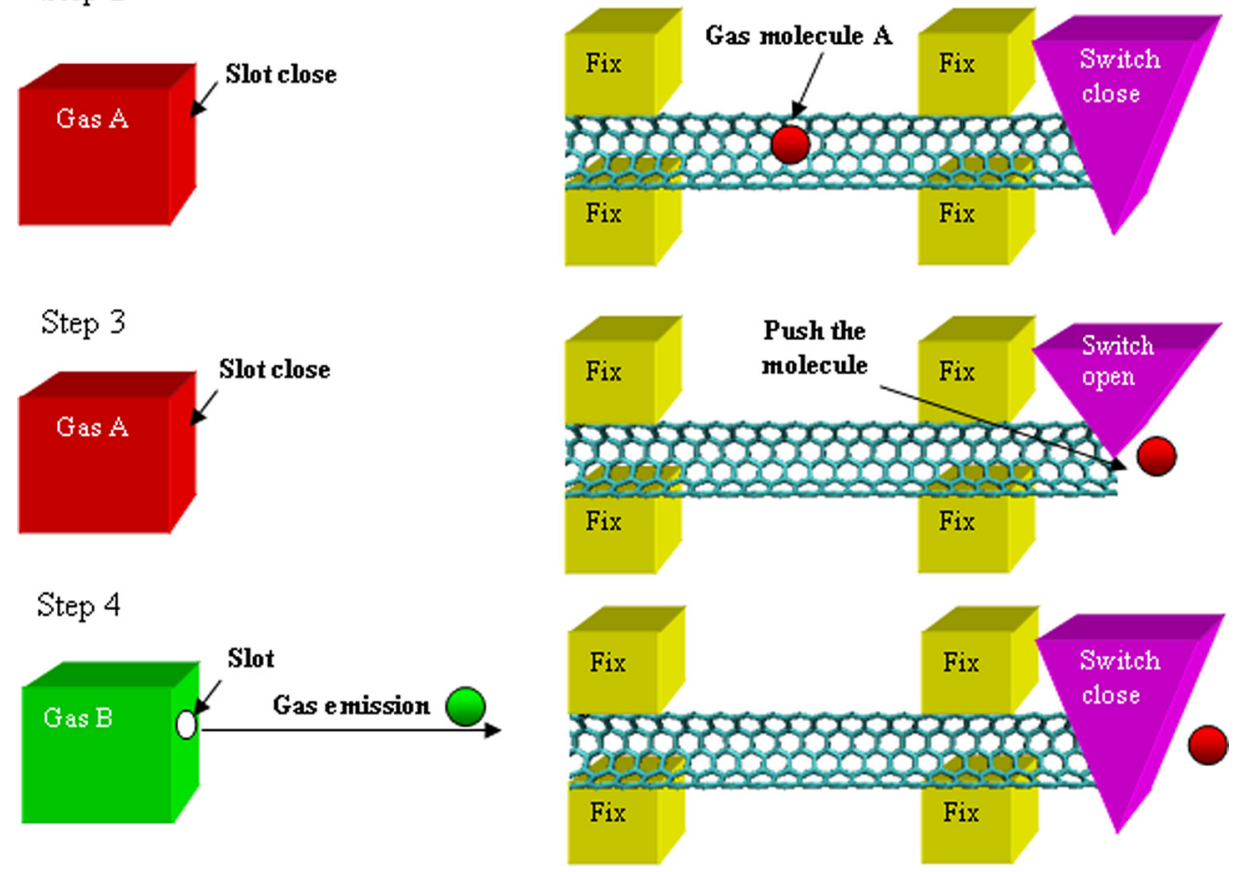

FIG. 1. Schematics of the encoding device. The encoding process can be realized by the execution of three steps. Step 1 shows the initial state of the system, in which a gas A is stored in a chamber with a slot. A small amount of gas A will be emitted from the slot and afterwards should be immediately pumped out. The right side of step 1 shows a schematic structure of a CNT resonator, which is placed in vacuum. The CNT is located via the two fixed positions and extends beyond the right fixing point. This extended length of the CNT will act as a storage space for the molecular chain. A switch is designed to the left side of this storage space. Step 2 shows that once the molecule is detected inside the CNT, the slot of the gas chamber will be shut down so as to interrupt the shooting before another molecule is inserted into the CNT. Step 3 illustrates the transportation of the molecule of gas A to the storage portion of the CNT which can possibly be done using methods such as temperature gradients (see Refs. 10 and 11). By repeating the above process (Step 4) with other kinds of gas molecule, while controlling the sequence of kind of gas, an encoded molecular chain may be obtained.

particle will still stay inside the CNT. After this, the switch (as showed in red part in Figure 2(a)) will be opened and methods such as temperature gradients ${ }^{10,11}$ can be used to transport the particle through the CNT to the storage area to the right of the switch. In accordance with the schematic shown in Figure 1, a molecular chain could be encoded.

Hereafter, encoder $\alpha$-was studied in an analogue fashion, which concluded that the amplitude decreased from $5.03 \AA$ to $1.65 \AA$ due to the insertion of a $\mathrm{Kr}$-atom, which once again verified that the particle is in the CNT. This change in the amplitude is much larger than that in encoder $\alpha+$, because of the nonlinearity of Duffing oscillator ${ }^{27}$ in encoder $\alpha+$ caused by excessively large amplitude.

For encoder $\beta+$, the molecular model is shown in Figure 2(c). Using a similar strategy as for the encoder $\alpha+$, the model reveals that the amplitude increased from $1.36 \AA$ to $6.95 \AA$ after the insertion of a $\mathrm{Kr}$-atom. This $\mathrm{Kr}$-atom was trapped near the CNT tip in the same way as for the $\alpha+$ encoder as shown in Figure 2(d). After this, a model particle with a mass $50 \%$ heavier than the $\mathrm{Kr}$-atom was shot into the $\mathrm{CNT}$, which has a previously trapped $\mathrm{Kr}$-atom. It revealed that the amplitude increased from $1.66 \AA$ to $7.35 \AA$ due to the insertion and the consequent trapping of the atom. Hence, a sample molecular chain of two molecules was encoded in this process.

Finally, encoder $\beta$ - is studied in the same way. In this analysis, the vibrational amplitude decreased from $6.29 \AA$ to $1.98 \AA$ after the insertion of a $\mathrm{Kr}$-atom. A further decrease from $6.95 \AA$ to $2.35 \AA$ is seen after another insertion of the model atom. Once again, a molecular chain is encoded.

The scheme proposed in Figure 1 is not only limited to an interesting theoretical exercise, but should also be studied from a practical viewpoint. The feasibility of the present work is examined in some detail in supplementary information Sec. B. ${ }^{30}$ To begin with, it should be ensured that the molecule can be shot into the CNT one at a time. In order to do this, the interval between the insertion of $\mathrm{Kr}$ gas molecule was estimated from Eq. (S1) of supplementary information Sec. B (Ref. 30) as $0.07 \mathrm{~ms}$, with a gas pressure of $1 \mathrm{~Pa}$, gas temperature $30 \mathrm{~K}$, and a $(5,5) \mathrm{CNT}$. This should be quite enough for steps 1-3 shown in Figure 1, let alone that in the laboratory condition the gas pressure can be several orders lower than $1 \mathrm{~Pa},{ }^{19}$ resulting in a much longer interval. The simulations reveal that the time for detection can be much shorter than the above-mentioned interval. Therefore, the possibility that more than one molecule will enter the CNT can be omitted by a fast shuttering of the slot. 
(a)

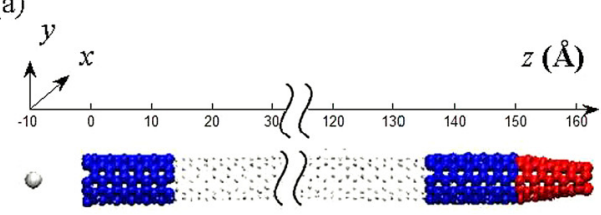

(b)

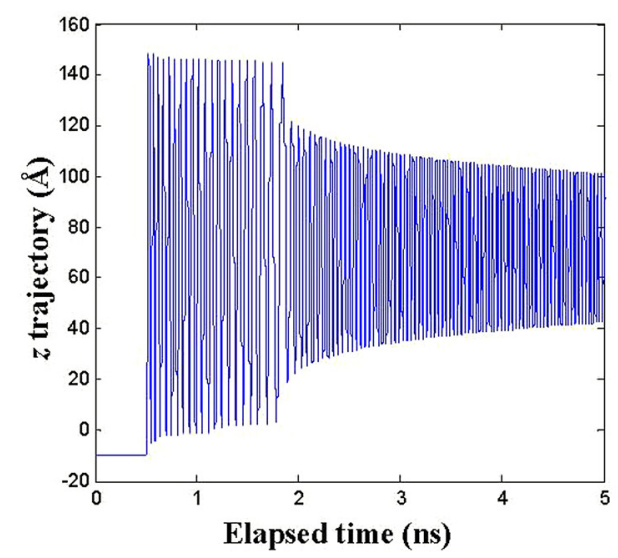

(c)

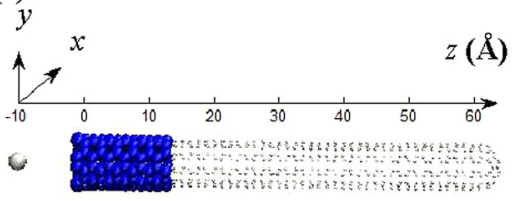

(d)

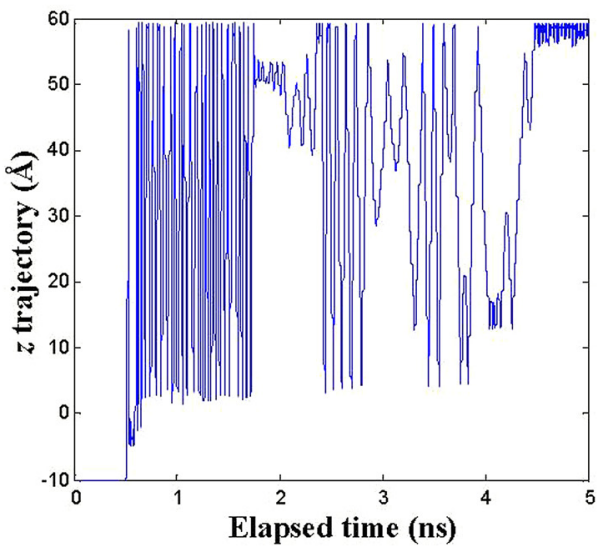

FIG. 2. Simulation information. (a) Initial state of " $\alpha$ " encoders at $t=2.5 \mathrm{ps}$. The white particle to the left is the Kr-atom with remaining part carbon. Blue atoms are clamped. The red part is under a pressure of $21 \mathrm{GPa}$ and therefore buckles radially in order to shut down the path as a switch. The area between the blue clamped positions is excited vibrationally. (b) Trapping of a Kr-atom expressed by its position in the $z$-direction as a function of time, during which an initially $>700 \mathrm{~m} / \mathrm{s}$ speed of the particle will gradually decrease and the particle will gradually approach the centre of the beam. According to inertia trapping, the particle will finally stay near the antinode of the CNT resonator, i.e. $z=70 \AA$. (c) the initial configuration for " $\beta$ " encoders, where the white particle to the left is the $\mathrm{Kr}$-atom with remaining part carbon. Blue atoms are clamped. The atoms to the right of the blue parts are vibrating. (d) Trapping of a Kr-atom expressed by its position in the $z$-direction as a function of time.

For " + " encoders, the process of inertia trapping should be verified. We then assessed from parameter $c$ in Eq. (S6) of supplementary information Sec. B (Ref. 30) that for either encoder $\beta+$ or $\alpha+$, the trapping is feasible. This parameter can be calculated as

$$
c=\sqrt{2 k_{B} T / \omega^{2} r_{p}^{2} m}
$$

where $k_{\mathrm{B}}$ is the Boltzmann constant, $T$ is the temperature of the system, $\omega$ is the angular frequency, $m$ is the mass of the particle, $r_{p}$ is the amplitude of particle's circular motion. If $c \ll 1$, there should be stable inertia trapping of the particle.

Another important aspect to be verified is the sensitivity of the CNT resonator with respect to the mass of the particle. This paper assessed that monatomic molecule, e.g., $\mathrm{Kr}$ or

TABLE I. A summary of main simulation results.

\begin{tabular}{lcccc}
\hline \hline Encoder $^{\mathrm{a}}$ & $F(\mathrm{GHz})^{\mathrm{b}}$ & $A^{0}(\AA)^{\mathrm{c}}$ & $A^{1}(\AA)^{\mathrm{c}}$ & $A^{2}(\AA)^{\mathrm{c}}$ \\
\hline$\alpha+$ & 157.2 & 8.80 & 8.99 & $\ldots$ \\
$\alpha-$ & 146.4 & 5.03 & 1.65 & $\ldots$ \\
$\beta-$ & 106.5 & 6.29 & 1.98 & $\ldots$ \\
$\beta-$ & 103.6 & $\ldots$ & 6.95 & 2.35 \\
$\beta+$ & 103.6 & 1.36 & 6.95 & $\ldots$ \\
$\beta+$ & 101.2 & $\ldots$ & 1.66 & 7.35 \\
\hline \hline
\end{tabular}

Type of encoder.

${ }^{b}$ Driving frequencies. They are some eigenfrequencies of the CNT with or without the particles. Detailed physical meaning is in supplementary material Sec. A. ${ }^{\mathrm{c}} A^{O}, A^{1}$, and $A^{2}$ are the amplitudes of vibration of the CNT with 0,1 , and 2 particles at the antinode, respectively. Notice that encoder $\alpha+$ and $\alpha-$ act differently with respect to activation. bio-molecules like nucleic acid should be detectable ${ }^{19-21}$ for two "+" encoders if good inertia trapping, as defined in supplementary information Sec. $\mathrm{B},{ }^{30}$ can be achieved. For the "-" encoders, the sensitivity would, however, be significantly lower.

Finally, to show the fabrication feasibility of the proposed method, we hereby present a possible design for " $\alpha$ " encoders as presented in Figure 3. The switch can be realized as either a silicon cantilever valve or based on electrostatic bending. Both solutions require the etching of a cavity below the CNT. Bending buckling, ${ }^{15}$ kinking $^{28}$ or perhaps other bucklings ${ }^{16}$ are thus planned for closing the path of the CNT since direct clamp flattening of the CNT is not likely. This

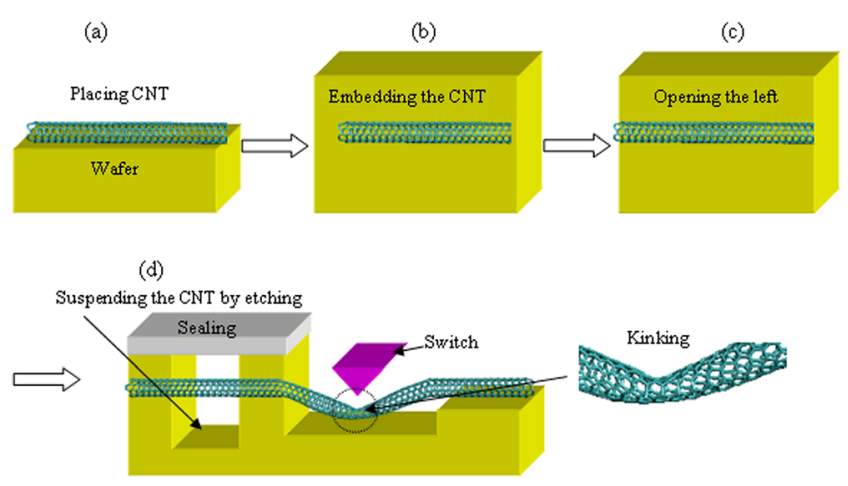

FIG. 3. A proposed fabrication process for " $\alpha$ " encoders. (a) Placing CNT on the wafer. (b) Depositing a Si layer to embed the CNT. (c) Opening the left by polishing/etching the wafer (see Ref. 23). (d) Suspending the CNT by etching to make the double-clamped beam (see Ref. 21), and to make the switch. The sealing is implemented due to the requirement of vacuum for the resonator. 
fabrication is readily available in any advanced clean room facility. After the molecular chain has been encoded, the verification of the product can possibly be done by observing the chain with a microscope ${ }^{29}$ in order to detect if the molecules have different shapes.

In summary, we show the detection of single gas molecules inside a CNT based on the change in resonance frequency and amplitude of the CNT associated with the inertia trapping phenomenon. As its direct implication, a method has been proposed to control molecular sequences inside a CNT. The method has been demonstrated by encoding a molecular chain inside a CNT with MD method. A theoretical assessment shows that it is possible that a physical model could be fabricated in the very near future in a suitable nanofabrication facility. There are several types of encoders. The analysis suggests that one should consider the $\beta+$ encoder, which requires lower driving frequencies, as a suitable starting point. The $\alpha+$ encoder that requires higher driving frequencies as well as the fabrication of the switch, due to the doubleclamped nature of the scheme, will perhaps be expensive, but worthy of our attention, due to the infinite length of the molecular chain that can thus be encoded. The "-" encoders are, however, not suitable due to their lower sensitivities.

The authors acknowledge Juan Atalaya and Andreas Isacsson, Department of Applied Physics, Chalmers University of Technology for useful discussions and input concerning the innovation of the manuscript. The computations were performed on C3SE computing resources at Chalmers University of Technology. This work was supported in part by the Chinese Ministry of Science and Technology through the international collaboration program with the contract No: 2010DFA14450, as well as by the EU programs "ThemaCNT," "Smartpower," and "Nanocom". This work was also carried out within the Sustainable Production Initiative and the Production Area of Advance at Chalmers. This support is gratefully acknowledged.

${ }^{1}$ G. Binnig and H. Rohrer, Helv. Phys. Acta. 55, 726 (1982).

${ }^{2}$ P. Avouris, Acc. Chem. Res. 28, 95 (1995).
${ }^{3}$ R. Feynman, Caltech Eng. Sci. 23(5), 22 (1960), available at http://resolver. caltech.edu/CaltechES:23.5.1960Bottom.

${ }^{4}$ J. Lee, W. Shen, K. Payer, T. P. Burg, and S. R. Manalis, Nano Lett. 10, 2537 (2010)

${ }^{5}$ Z. Hu, G. Mårtensson, M. Murali, X. Guo, and J. Liu, "Atomic scale mass transport based on inertial trapping in SWCNT resonators," J. Appl. Phys. (submitted).

${ }^{6}$ J. Atalaya, A. Isacsson, and M. I. Dykman, Phys. Rev. Lett. 106, 227202 (2011).

${ }^{7}$ K. Kim, K. Jensen, and A. Zettl, Nano Lett. 9, 3209 (2009).

${ }^{8}$ S. Regan, R. O. Aloni, U. D. Ritchie, and A. Zettl, Nature 428, 924 (2004).

${ }^{9}$ H. Liu, J. He, J. Tang, H. Liu, P. Pang, D. Cao, P. Krstic, S. Joseph, S. Lindsay, and C. Nuckolls, Science 327, 64 (2010).

${ }^{10}$ P. A. E. Schoen, J. H. Walther, D. Poulikakos, and P. Koumoutsakos, Appl. Phys. Lett. 90, 253116 (2007).

${ }^{11}$ A. Barreiro, R. Rurali, E. R. Hernández, J. Moser, T. Pichler, L. Forró, and A. Bachtold, Science 320, 775 (2008).

${ }^{12}$ H. Qiu, R. Shen, and W. Guo, Nano Res. 4, 284 (2011).

${ }^{13}$ B. Li and T. Chang, J. Appl. Phys. 108, 054304 (2010).

${ }^{14}$ T. Chang, Phys. Rev. Lett. 101, 175501 (2008).

${ }^{15}$ Z. Hu, X. Guo, and J. Liu, J. Comput. Theor. Nanosci. 8, 1716 (2011).

${ }^{16} \mathrm{Q}$. Wang, Nano Lett. 9, 245 (2009).

${ }^{17}$ J. Chaste, M. Sledzinska, M. Zdrojek, J. Moser, and A. Bachtold, Appl. Phys. Lett. 99, 213502 (2011).

${ }^{18}$ E. A. Laird, F. Pei, W. Tang, G. A. Steele, and L. P. Kouwenhoven, Nano Lett. 12, 193 (2012).

${ }^{19}$ K. Jensen, K. Kim, and A. Zettl, Nat. Nanotechnol. 3, 533 (2008).

${ }^{20}$ A. K. Huttel, G. A. Steele, B.Witkamp, M. Poot, L. P. Kouwenhoven, and H. S. J. van der Zant, Nano Lett. 9, 2547 (2009).

${ }^{21}$ J. Chaste, A. Eichler, J. Moser, G. Ceballos, R. Rurali, and A. Bachtold, Nat. Nanotechnol. 7, 301 (2012).

${ }^{22}$ K. Jensen, J. Weldon, H. Garcia, and A. Zettl, Nano Lett. 7, 3508 (2007).

${ }^{23}$ C. Y. Lee, W. Choi, J. H. Han, and M. S. Strano, Science 329, 1320 (2010).

${ }^{24}$ D. G. Gibson, J. I. Glass, C. Lartigue, V. N. Noskov, R. Y.Chuang, M. A. Algire, G. A. Benders, M. G. Montague, L. Ma, M. M. Moodie, and C. Merryman et al., Science 329, 52 (2010).

${ }^{25}$ M. A. Lantz, B.Gotsmann, U. T. Dürig, P. Vettiger, Y. Nakayama, T. Shimizu, and H. Tokumoto, Appl. Phys. Lett. 83, 1266 (2003).

${ }^{26}$ E. Bichoutskaia, A. M. Popov, and Y. E. Lozovik, Mater. Today 11, 38 (2008).

${ }^{27}$ G. Duffing, Erzwungene Schwingungen bei Veränderlicher Eigenfrequenz (F. Vieweg u. Sohn, Braunschweig, 1918).

${ }^{28}$ D. Qian, G. J. Wagner, W. K. Liu, M. F. Yu, and R. S. Ruoff, Appl. Mech. Rev. 55, 495 (2002).

${ }^{29}$ K. Suenaga, M. Tencé, C. Mory, C. Colliex, H. Kato, T. Okazaki, H. Shinohara, K. Hirahara, S. Bandow, and S. Iijima, Science 290, 2280 (2000).

${ }^{30}$ See supplementary material at http://dx.doi.org/10.1063/1.4754617 for MD simulation and feasibility analysis. 